\title{
Gender, hormone therapy, and HIV: what should cardiologists know?
}

\author{
C. A. Martinez · R. R. Rikhi
}

Published online: 22 January 2019

(C) The Author(s) 2019

\begin{abstract}
Transgender individuals represent the fastest growing minority in the United States and are disproportionately affected by HIV. Hormone therapy is the most common treatment for gender dysphoria in transgender individuals. As HIV is an independent risk factor for coronary artery disease, it is critical to further research the influence masculinising and feminising hormone therapies have on cardiovascular disease. There is a clinical need for evidencebased guidelines for cardiologists to follow to effectively care for and treat transgender patients. For this to be done, the interplay between HIV, hormone therapy, and cardiovascular disease must be better understood through collaboration between researchers and clinicians to achieve maximum benefit from recent advancements.
\end{abstract}

Keywords Cardiovascular disease $\cdot$ Hormone replacement therapy $\cdot$ Transgender persons $\cdot$ HIV

\section{Introduction}

Biological sex has been recognised as an independent risk factor for the development of cardiovascular disease (CVD) [1]. In recent years, there has been an increased awareness regarding the spectrum of gender diversity and the role of gender as a CVD risk factor, independent of biological sex [1]. Transgender is an overarching term to describe individuals whose gender identity is different from their sex assigned at birth [1]. They play an integral role within the spectrum of gender diversity and are becoming the fastest growing minority in the United States, with more than 1.4 mil-

C. A. Martinez $(\bowtie) \cdot$ R. R. Rikhi

Department of Medicine, Miller School of Medicine,

University of Miami, Miami, FL, USA

cmartinez5@med.miami.edu lion individuals identifying as transgender [2]. There is a growing body of research investigating health disparities in this population; yet, limited information exists regarding the cardiovascular risk profiles of transgender men and women. Thus, current cardiac risk stratification tools are complicated and inaccurate because the confounding effect of exogenous hormone therapy on biological sex-based cardiac risk calculators is unknown [1]. This is highly concerning for the current medical society, since there are limited evidence-based recommendations to guide management for CVD prevention in the transgender community.

There are two relevant aspects that should be recognised when providing care for transgender individuals: (1) more than $75 \%$ of transgender men and women are on either masculinising or feminising hormone therapy, respectively, and (2) they are disproportionately affected by the HIV epidemic [1]. Although HIV is an important risk factor for the development of CVD, there is a controversial understanding of the cardiovascular effects of concomitant hormone therapy.

\section{Hormones and CVD at a cellular level}

Seminal research has focused on understanding the cellular interaction of masculinising and feminising hormones on the vasculature. Endogenous hormones directly influence vascular endothelial function through androgen receptors (ARs) and oestrogen receptors (ERs) present on endothelial cells [3]. After ligand binding, oestrogen and testosterone translocate into the nuclei of endothelial cells, leading to genomic effects [4]. Under physiological conditions, endogenous activation of both ARs and ERs leads to upregulation of athero-protective genes and downregulation of pro-atherogenic genes [4]. Addition- 
ally, activation of both receptors has been shown to have non-genomic effects, by directly upregulating endothelial nitric oxide synthesis, which mediates vasodilatory function [3]. However, these effects have not been translated into clinical outcomes.

\section{Hormones and CVD in cisgender individuals}

Despite the expansive use of hormone therapy in cisgender individuals, conflicting evidence still exists regarding their cardiovascular effects [4]. Premenopausal women have a lower incidence of coronary artery disease than age-matched cisgender men and postmenopausal women, suggesting the cardioprotective effects of endogenous oestrogen [4]. However, the effects of oestrogen replacement therapy continue to be controversial. The Women's Health Initiative Hormone Therapy Trials found an 18\% increase in coronary heart disease in the oestrogen plus progestin group and no difference in the oestrogen only group, when compared to placebo [5]. In contrast, longer follow-up of the younger participants in this trial revealed that oestrogen therapy improved atherosclerosis outcomes [5]. Data on the effect of hormone therapy in cisgender men also suggest an elevated CVD risk. Although testosterone replacement therapy has been used to treat hypogonadism in men, animal and human studies have shown that testosterone treatment for more than 12 weeks increases cardiovascular morbidity and mortality [6]. Testosterone use for more than 1 year in cisgender men has also been associated with increased coronary artery plaque volume when compared to placebo [7]. Nevertheless, data from these trials are not applicable to transgender individuals receiving feminising or masculinising hormone therapy because the clinical characteristics of the study population and hormone regimens differ vastly [1].

\section{Hormones and CVD in transgender individuals}

Many transgender individuals seek long-term hormone therapy to promote physical characteristics that align with their gender identity and to suppress effects of endogenous hormones that are discordant to their identified gender [6]. To date, very few studies have examined the cardiovascular effects of masculinising and feminising hormones in transgender individuals; and those that exist are mired in contradictory results. Masculinising and feminising hormones have been reported to both impair and improve several cardiovascular risk markers [6]. A few studies examining cardiovascular risk among transgender individuals have indicated an increase in hypertension, dyslipidaemia, and diabetes with longterm hormone therapy $[6,8]$. A retrospective study in transgender women suggested an increase in cardiovascular morbidity and mortality; however, this has not been demonstrated for transgender men $[1,6$, 8]. In addition, these studies are mainly retrospective with several limitations, such as not controlling for smoking or other CVD variables, as well as lacking
Fig. 1 Transgender individuals are disproportionately affected by HIV and an increasing number are exposed to hormone therapy. The cardiovascular effects of HIV and hormone therapy among transgender individuals are not known
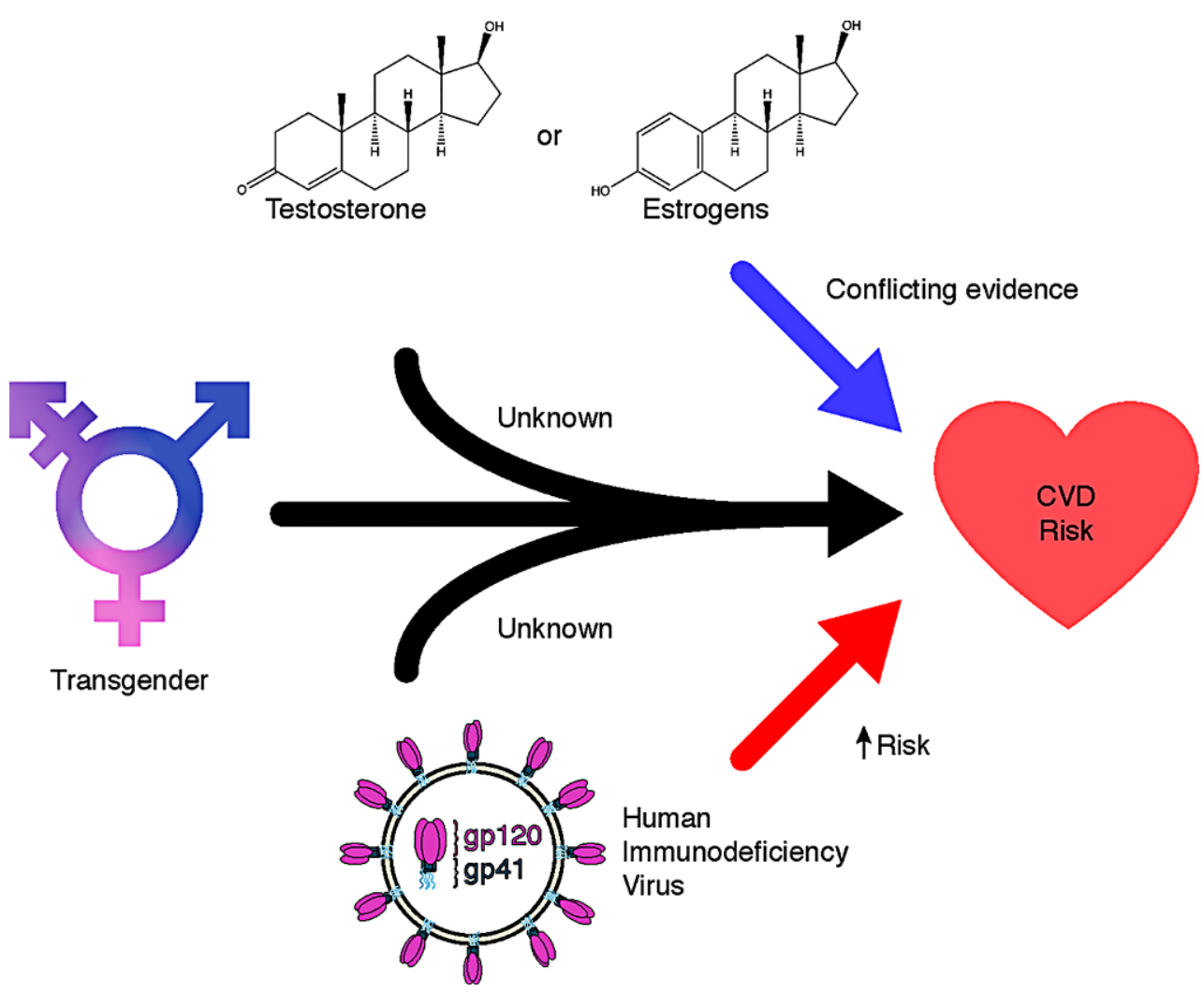
transgender control groups [1]. In view of the limited available evidence, current guidelines for the primary and gender-affirming care of transgender and gender non-binary people state that CVD risk is unchanged in transgender men, and that the evidence is less clear for transgender women [1].

\section{HIV and CVD}

$\mathrm{HIV}$ is now recognised as a CVD risk factor, as it causes immune dysfunction and a heightened inflammatory state that accelerates the development of CVD pathogenesis [9, 10]. Transgender individuals are the highest at-risk population for HIV infection [1]. With the advent of antiretroviral medication therapy, all HIV-infected individuals are living longer and, consequently, have an increased risk for cardiovascular complications [11]. However, HIV-infected persons have elevated subclinical cardiometabolic complications occurring 10-15 years in advance compared to seronegative counterparts $[9,10]$. These subclinical cardiometabolic complications include central adiposity, dyslipidaemia, glucose intolerance, and hypertension [9]. There is conflicting evidence regarding primary and secondary prevention guidelines for HIV-infected individuals [12]. In part, this is due to limited information regarding interactions between cardiovascular preventative medications (i.e. antiplatelet and lipid-lowering medications) and antiretroviral therapies [12]. Further, studies have shown that dyslipidaemia may independently interact with HIV to increase inflammation [12]. Biological sex has been shown to impact the effect of HIV on CVD, as studies have shown biological females with HIV to have twice the risk of CVD compared to biological males with HIV [10]. Of great concern is that transgender men and women living with HIV have higher rates of overall morbidity and earlier mortality compared to cisgender HIV-infected individuals [2]. It is not known if CVD risk contributes to this health disparity.

\section{HIV, hormones, and CVD}

There is a paucity of evidence indicating whether HIVrelated CVD risk differs in transgender individuals prescribed hormone therapy (Fig. 1). Therefore, a greater awareness of the potential to modify cardiometabolic risk factors associated with both HIV and hormone therapy is encouraged, as it will help decrease the potential CVD burden in this population [9].

\section{Current treatment}

While there are ongoing studies on primary prevention in patients with HIV [13, 14], there are no studies focussing specifically on cardiovascular prevention among transgender persons with HIV. However, there are certain strategies that can be implemented and are applicable to this population, such as encouraging smoking cessation, lipid monitoring and appropriate treatment with a statin that has limited drug interactions with antiretroviral therapy, appropriate selection of antiplatelet therapy, maintaining blood pressure and glucose control, and regular exercise and optimal body mass index. In addition, hormone therapy regimens with the least cardiovascular adverse effects should be used and hormone levels should be monitored to avoid supra-physiological levels that may be associated with a higher CVD risk [15].

\section{Conclusion}

A large gap exists in our understanding of how to effectively provide evidence-based care for transgender individuals. This is a vulnerable population that not only faces constantly shifting social policies, but also clinical challenges due to the complex interplay of CVD risk factors, HIV, and hormone therapy. A multidisciplinary team approach must focus on addressing unique health care needs among the transgender community. Clinicians together with researchers must join to help narrow this gap and obtain answers that will help develop public health interventions specifically for this medically underserved population.

Acknowledgements We would like to acknowledge Jaret M. Karnuta, B.S. for assisting in the creation of Fig. 1.

Funding This work was supported by the authors. We received no grants or funding.

Conflict of interest C.A. Martinez and R.R. Rikhi declare that they have no competing interests.

Open Access This article is distributed under the terms of the Creative Commons Attribution 4.0 International License (http://creativecommons.org/licenses/by/4.0/), which permits unrestricted use, distribution, and reproduction in any medium, provided you give appropriate credit to the original author(s) and the source, provide a link to the Creative Commons license, and indicate if changes were made.

\section{References}

1. Deutsch M. Guidelines for the primary and gender-affirming care of transgender and gender nonbinary people. San Francisco: Center of Excellencefor Transgender Health, Department of Family and Community Medicine, University ofCalifornia; 2016.

2. Mayer KH, Grinsztejn B, El-Sadr WM. Transgender people and HIV prevention: what we know and what we need to know, a call to action. J Acquir Immune Defic Syndr. 2016;72:207-9.

3. Morselli E, Santos RS, Criollo A, et al. The effects of oestrogens and their receptors on cardiometabolic health. NatRevEndocrinol. 2017;13:352-64.

4. Herrmann JL, Abarbanell AM, Weil BR, et al. Gender dimorphisms in progenitor and stem cell function in cardiovascular disease. J Cardiovasc Transl Res. 2010;3:103-13.

5. MansonJE, Chlebowski RT, StefanickML, etal. Menopausal hormone therapy and health outcomes during the intervention and extended poststopping phases of the 
Women's Health Initiative randomized trials. JAMA. 2013;310:1353-68.

6. Streed CGJr., Harfouch O, MarvelF, et al. Cardiovascular diseaseamong transgender adults receivinghormonetherapy: a narrative review. Ann Intern Med. 2017;167:256-67.

7. Xu L, Freeman G, Cowling BJ, et al. Testosterone therapy and cardiovascular events among men: a systematic review andmeta-analysis of placebo-controlledrandomized trials. BMCMed. 2013;11:108.

8. Maraka S, Singh Ospina N, Rodriguez-Gutierrez R, et al. Sex steroids and cardiovascular outcomes in transgender individuals: a systematic review and meta-analysis. J Clin Endocrinol Metab. 2017;102:3914-23.

9. Havlir DV, Currier JS. CROI 2016: complications of HIV infection and antiretroviral therapy. Top Antivir Med. 2016;24:38-46.

10. Vachiat A, McCutcheon K, Tsabedze N, et al. HIV and ischemic heart disease. JAm Coll Cardiol. 2017;69:73-82.

11. Petoumenos K, Worm SW. HIV infection, aging and cardiovascular disease: epidemiology and prevention. SexHealth. 2011;8:465-73.
12. Demir OM, Candilio L, Fuster D, et al. Cardiovascular disease burden among human immunodeficiency virusinfected individuals. Int J Cardiol. 2018;265:195-203.

13. Gilbert JM, Fitch KV, Grinspoon SK. HIV-related cardiovascular disease, statins, and the REPRIEVE trial. Top Antivir Med. 2015;23:146-9.

14. Aberg JA, Sponseller CA, Ward DJ, et al. Pitavastatin versus pravastatin in adults with HIV-1 infection and dyslipidaemia (INTREPID): 12 week and 52 week results of a phase 4 , multicentre, randomised, double-blind, superiority trial. Lancet HIV. 2017;4:e284-e94.

15. Van Horn L, Carson JA, Appel LJ, et al. Recommended dietary pattern to achieve adherence to the American Heart Association/American College of Cardiology (AHA/ACC) guidelines: a scientific statement from the American Heart Association. Circulation. 2016;134:e505-e29. 